\title{
Composição florística e estrutura do componente arbóreo de uma floresta altimontana no município de Camanducaia, Minas Gerais, Brasil
}

\author{
GLAUCO S. FRANÇA ${ }^{1}$ e JOÃO R. STEHMANN ${ }^{2,3}$
}

(recebido: 26 de dezembro de 2002; aceito: 9 de outubro de 2003)

\begin{abstract}
Floristic composition and structure of a tree component of a high montane forest in the municipality of Camanducaia, Minas Gerais, Brazil). The floristic composition and phytosociological structure were investigated in an area of 0.75 ha of a montane forest (1,900 m a.s.l.) located between the municipality of Gonçalves and Camanducaia in the Serra da Mantiqueira. This forest belongs to the largest forest remnant in the region, and keep several headwaters of rivers which waters supply many cities in southern Minas Gerais and São Paulo. The phytosociological survey was done on 15 plots of 5 $\times 100 \mathrm{~m}$ where all trees with $\mathrm{DBH} \geq 15 \mathrm{~cm}$ were recorded. The floristic survey listed 66 species and 29 families. The families with highest species richness were Myrtaceae and Solanaceae. The phytosociological survey recorded 1,501 individuals in 58 espécies, with density of 2,001 individuals per hectare. The most important families were Myrtaceae, Lauraceae and Meliaceae. The species with higher IVI were Ocotea lancifolia (42.88), Cabralea canjerana (29.53) and Psychotria velloziana (22.95). Species diversity $\left(\mathrm{H}^{\prime}\right)$ was estimated as 2.9 (nats.ind. ${ }^{-1}$ ). The floristic inventary showed an important richeness with typical relictual montane species and the presence of an endangered species, Dicksonia sellowiana (Dicksoniaceae). The protection of this remnant is very important as strategy of biodiversity conservation of atlantic tropical montane forest and as water resource to the southern Minas Gerais and São Paulo.
\end{abstract}

Key words - floristic composition, phytosociological structure, tropical montane forest

RESUMO - (Composição florística e estrutura do componente arbóreo de uma floresta altimontana no município de Camanducaia, Minas Gerais, Brasil). Investigou-se a composição florística e a estrutura fitossociológica em 0,75 ha de floresta altimontana, a 1.900 m s.n.m., localizada na divisa dos municípios de Camanducaia e Gonçalves, na Serra da Mantiqueira. Essa área situa-se no maior remanescente de mata da região, que abriga diversas nascentes de rios cujas águas abastecem várias cidades do sul de Minas Gerais e de São Paulo. O levantamento fitossociológico foi realizado em 15 parcelas de $5 \times 100 \mathrm{~m}$, onde todos os indivíduos com circunferência do tronco à altura do peito $($ ca. $1,30 \mathrm{~m}) \geq 15 \mathrm{~cm}$ foram registrados, tendo seus diâmetros medidos e alturas estimadas. O levantamento florístico registrou a presença de 66 espécies arbóreas pertencentes a 29 famílias, muitas delas típicas de formações florestais de altitude do sul e sudeste do Brasil, bem como uma espécie ameaçada de extinção, Dicksonia sellowiana (Dicksoniaceae). As famílias com maior riqueza em espécies foram Myrtaceae e Solanaceae. No levantamento fitossociológico foram amostrados 1.501 indivíduos distribuídos em 58 espécies e 29 famílias. A densidade total calculada foi de 2.001 indivíduos por hectare. As famílias mais importantes foram Myrtaceae, Lauraceae e Meliaceae. As espécies com maior IVI foram Ocotea lancifolia $(42,88)$, Cabralea canjerana $(29,53)$ e Psychotria velloziana $(22,95)$. O índice de diversidade (H') foi de 2,9 (nats.ind. ${ }^{-1}$ ). A proteção deste remanescente é de fundamental importância para a conservação da biodiversidade da floresta atlântica de altitude e dos recursos hídricos da região sul de Minas Gerais.

Palavras-chave - composição florística, estrutura fitossociológica, floresta tropical montana

\section{Introdução}

As florestas montanas são reconhecidamente muito pouco estudadas e estão entre as mais ameaçadas de todas as vegetações florestais dos trópicos (Gentry 1995). Elas apresentam uma elevada biomassa de briófitas, uma alta riqueza em espécies de pteridófitas e

1. Parte da dissertação de mestrado - Pós-Graduação em Biologia Vegetal, ICB, UFMG.

2. Universidade Federal de Minas Gerais, Instituto de Ciências Biológicas, Departamento de Botânica, Av. Antônio Carlos 6627, 31270-110 Belo Horizonte, MG, Brasil.

3. Autor para correspondência: stehmann@icb.ufmg.br uma menor riqueza de angiospermas em relação às florestas localizadas em baixas altitudes (Webster 1995). Essa diversidade de espécies permite fisionomias e estruturas florestais que se sucedem num gradiente montanha acima, diferenciando-se normalmente a curtas distâncias (Whitmore 1990).

No Brasil, as florestas de altitude ou montanas (sensu lato) ocorrem no alto dos planaltos ou serras, atingem até $2.200 \mathrm{~m}$ s.n.m. e têm sido subdivididas em montanas (sensu stricto) e altimontanas, distribuindo-se em diferentes faixas de altitude dependendo da latitude. Na região sudeste do Brasil, as florestas montanas estão situadas entre $16^{\circ}$ e $24^{\circ}$ de latitude $\mathrm{S}$, ocorrendo em altitudes de $500 \mathrm{~m}$ até $1.500 \mathrm{~m}$. As florestas altimontanas 
são encontradas acima dos limites estabelecidos para a formação montana (Veloso et al. 1991, Oliveira Filho \& Fontes 2000). Nesta tipologia encontram-se incluídas também as florestas nebulares ou "matas nuvígenas", localizadas no cume das altas montanhas, geralmente acima de $2.000 \mathrm{~m}$ de altitude, como aquelas encontradas na Serra do Itatiaia (Segadas-Vianna 1968). No sul do Brasil, as florestas nebulares ocorrem em altitudes menores (700-1.600 m), ocupando as altas montanhas do complexo da Serra do Mar e a borda do planalto da Serra Geral (Falkemberg \& Voltolini 1995).

A literatura conta com poucos levantamentos florísticos de vegetação florestal acima dos $1.000 \mathrm{~m}$ s.n.m. No Brasil, a maioria foi realizada em florestas montanas (s. str.) brasileiras do estado de São Paulo (Pagano \& Leitão Filho 1987). Para a região sudeste podemos citar os trabalhos na Serra de São José, MG (Oliveira Filho \& Machado 1993), na Reserva Biológica Poço Bonito, MG (Oliveira Filho et al. 1994a), no Parque Estadual do Ibitipoca, MG (Fontes 1997, Carvalho et al. 2000), na Serra do Cipó, MG (Meguro et al. 1996), na Reserva Ecológica de Macaé de Cima, RJ (Guedes-Bruni et al. 1997), no Parque Municipal da Grota Funda, SP (Grombone et al. 1990) e na Serra do Japi, SP (Rodrigues et al. 1989).

No Sul de Minas Gerais, a cobertura vegetal original era constituída por extensas formações florestais montanas (s. lato), caracterizadas por comunidades arbóreas mais ou menos densas, com indivíduos pouco ramificados na porção média e inferior do tronco, e formações campestres representadas pela Savana Campos, Savana arborizada - Cerrado e Savana especial dos altos divisores (Azevedo 1962). As regiões florestadas corresponderiam às seguintes tipologias: Floresta Estacional Semidecidual, Floresta Ombrófila Densa e Floresta Ombrófila Mista (Veloso et al. 1991). Nas últimas décadas este contínuo de vegetação foi reduzido a poucos remanescentes que se encontram atualmente em diferentes estádios de regeneração.

Um dos maiores remanescentes de floresta montana (s. lato) no sul de Minas Gerais encontra-se localizado nesta serra, mais especificamente na Área de Proteção Ambiental Fernão Dias (APA Fernão Dias) e é de extrema importância ambiental, pois abriga as principais nascentes dos rios Capivari, Sapucaí-Mirim, Jaguari e Camanducaia, cujas águas abastecem várias cidades do sul de Minas Gerais e o pólo industrial da região metropolitana de São Paulo.

Embora pouco estudada, a região da serra da Mantiqueira foi recentemente considerada área de importância biológica especial e prioritária para a conservação da biodiversidade em Minas Gerais, devido à alta riqueza de espécies da fauna e flora e a presença de endemismos (Costa et al. 1998).

O objetivo do presente trabalho foi estudar este remanescente, através do inventário florístico e da descrição do seu componente arbóreo, e compará-lo com outras florestas de altitude do sudeste do Brasil.

\section{Material e métodos}

O local estudado situa-se próximo à nascente do Rio Camanducaia, entre os municípios de Camanducaia e

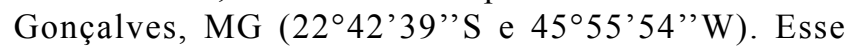
remanescente, conhecido como "Matão", possui aproximadamente 5.000 ha, e faz parte do Complexo da Serra da Mantiqueira. Para a localização das parcelas, escolheu-se a área mais plana e alta do remanescente, em uma altitude de cerca de 1.900 m s.n.m. Dentro da classificação proposta por Veloso et al. (1991), a mata enquadra-se na tipologia Floresta Ombrófila Densa Altimontana, embora floristicamente possua elementos da Floresta Ombrófila Mista.

A floresta está em bom estado de conservação e não se observam vestígios recentes de exploração seletiva de madeira. A maior perturbação é decorrente da presença do gado na floresta em algumas épocas do ano, formando trilhas e afetando a vegetação do sub-bosque. A borda da floresta é circundada de pastagens e culturas anuais. Existem relatos históricos de queimadas ocorridas há mais de 60 anos.

A região apresenta um clima do tipo Cwbl na classificação de Köppen (temperado, com verão fresco que se verifica até nas serras elevadas da zona tropical e valores médios de temperatura entre $10^{\circ} \mathrm{C}$ e $22^{\circ} \mathrm{C}$ - INMET 1982). Os dados fornecidos pelo 5을 Distrito de Meteorologia (5 DISME-

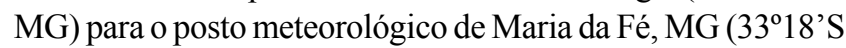
e $56^{\circ} 23^{\prime} \mathrm{W}, 1.200 \mathrm{~m}$ s.n.m.), no período de janeiro de 1991 a fevereiro de 2001, mostram valores médios de temperatura de $19^{\circ} \mathrm{C}$. Esta estação foi escolhida pela proximidade com a área de estudo, embora sua altitude seja $700 \mathrm{~m}$ menor e em latitude diferente, o que faz necessário ajustar aproximadamente menos $4{ }^{\circ} \mathrm{C}$ para os valores de temperatura (Walter 1986). A precipitação anual média foi de $1.417 \mathrm{~mm}$, sazonalmente distribuída. As chuvas estão concentradas no verão, nos meses de dezembro, janeiro e fevereiro, e o inverno registra déficit hídrico, especialmente nos meses de julho e agosto. Tal fato, contudo, parece não afetar significativamente a fisionomia da floresta, e a caducifolia, ainda que presente, é menos marcante que aquela encontrada em outras Florestas Estacionais Semideciduais Montanas do sul de Minas Gerais.

No município de Camanducaia as altitudes variam de 1.100 a 2.100 m s.n.m. O relevo da região é fortemente ondulado, com vales profundos nas zonas de drenagem dos rios que nascem na área do remanescente estudado. Os solos da região foram analisados e classificados utilizando-se o Sistema Brasileiro de Classificação de Solos (Embrapa 1999) 
e a Classificação de Solos do Brasil (Oliveira 1992) e o Mapa de Solos do Brasil, escala 1:5.000.000 (Embrapa 1981). Predominaram o Latossolo Vermelho-Amarelo Distrófico textura média e o Organossolo com acúmulo de turfeiras nas regiões florestadas. Quanto à granulometria, verificou-se uma alta porcentagem de areia, média de argila e baixa de silte, caracterizando-o como franco arenoso. A análise química indicou um solo fortemente ácido $(\mathrm{pH}=4,4)$. Foram verificadas altas taxas de $\mathrm{Al}\left(3,64 \mathrm{cmolc} \cdot \mathrm{dm}^{-3}\right), \mathrm{C}\left(8,08 \mathrm{dag} \cdot \mathrm{kg}^{-1}\right)$ e $\mathrm{N}$ $\left(0,63\right.$ dag. $\left.\mathrm{kg}^{-1}\right)$, além de elevada quantidade de matéria orgânica $\left(13,92\right.$ dag. $\left.\mathrm{kg}^{-1}\right)$.

Para a realização do levantamento florístico, espécimes férteis foram coletados aleatoriamente no período de julho de 2000 a julho de 2001. O material botânico foi herborizado (Fidalgo \& Bononi 1984) e incorporado ao Herbário BHCB, do Departamento de Botânica do Instituto de Ciências Biológicas da Universidade Federal de Minas Gerais (UFMG). As identificações foram feitas por comparação com espécimes depositados nas coleções dos herbários BHCB, IAC e UEC, além de consultas à literatura pertinente e envio de duplicatas para especialistas em instituições nacionais e estrangeiras.

No levantamento fitossociológico foram marcadas 15 parcelas $(5 \times 100 \mathrm{~m})$, totalizando $0,75 \mathrm{ha}$, distribuídas de forma perpendicular a dois transectos, de $200 \mathrm{~m}$ cada, localizados próximos à nascente do rio Camanducaia. Foram amostrados os indivíduos arbóreos com CAP (circunferência do caule à altura do peito) $\geq 15 \mathrm{~cm}$, a $1,30 \mathrm{~m}$ do solo, sendo todos eles etiquetados com plaquetas de alumínio numeradas. A altura das árvores foi estimada visualmente com o auxílio do podão de coleta. Os indivíduos amostrados foram coletados em fase reprodutiva e/ou vegetativa, sendo herborizados e posteriormente identificados. Foram calculados os parâmetros fitossociológicos de densidade, freqüência, dominância e valor de importância (Magurran 1988a). Calculou-se, ainda, o índice de diversidade de Shannon-Wiener (H') e o índice de equabilidade de Pielou (J'). Utilizou-se, para esses cálculos, o programa Fitopac (Shepherd 1994).

Para as comparações florísticas foram selecionados alguns trabalhos realizados em florestas montanas (s.lato) nos estados de Minas Gerais, São Paulo e Rio de Janeiro. A similaridade florística foi calculada através do coeficiente de Jaccard (Magurran 1988b).

\section{Resultados}

No levantamento florístico foram registradas 70 espécies arbóreas pertencentes a 29 famílias (tabela 1). As famílias com maior número de espécies no levantamento total foram: Myrtaceae (12), Asteraceae (9), Melastomataceae (5), Rubiaceae (5) e Solanaceae (5). Os gêneros com maior número de espécies foram: Myrceugenia (5), Solanum (4), Miconia, Psychotria e Symplocos (3).
Para o levantamento fitossociológico, foram amostrados nas 15 parcelas (0,75 ha), 1.501 indivíduos, dos quais 1.378 eram árvores vivas e 124, mortas ainda de pé. Os indivíduos vivos amostrados pertencem a 58 espécies (tabela 3) e 29 famílias, resultando em uma densidade estimada de 2.001 indivíduos.ha ${ }^{-1}$. A área basal foi estimada em $48,12 \mathrm{~m}^{2} \cdot \mathrm{ha}^{-1}$. O índice de diversidade de Shannon-Wiener ( $\mathrm{H}^{\prime}$ ) foi de 2,9 nats.indivíduo ${ }^{-1} \mathrm{e} o$ índice de equabilidade de Pielou (J) foi 0,71.

As famílias com maior número de indivíduos foram: Myrtaceae (326), Meliaceae (174), Winteraceae (169), Rubiaceae (164), Lauraceae (138), Melastomataceae (129), Myrsinaceae (94) e Monimiaceae (49). Juntas, estas famílias totalizaram $71,69 \%$ dos indivíduos marcados.

Quanto ao IVI (índice de valor de importância) destacaram-se: Myrtaceae $(54,17)$, Lauraceae $(46,09)$, Meliaceae $(29,52)$, Rubiaceae $(24,58)$ e Winteraceae $(18,16)$. Os maiores valores de IVI de Myrtaceae pertenceram a Myrcia fallax $(18,8)$, Myrceugenia alpigena $(9,42)$ e Myrcia venulosa. $(9,34)$. O maior IVI de Lauraceae pertenceu a Ocotea lancifolia $(42,88)$, o de Meliaceae, a Cabralea canjerana $(29,52)$ e o de Rubiaceae, a Psychotria velloziana $(22,95)$. Dessa forma, oito famílias contribuíram com cerca de $72 \%$ do IVI total.

As famílias com maior riqueza de espécies foram: Myrtaceae (11), Solanaceae (6), Lauraceae (4), Rubiaceae (3), Symplocaceae (3) e Asteraceae (3). Juntas elas representaram $51,72 \%$ do total de espécies levantadas.

A mata estudada possui um dossel alto e uniforme, sem espécies emergentes, com árvores atingindo até $22 \mathrm{~m}$. A estratificação é difícil de ser delimitada, mas grosseiramente podemos reconhecer três estratos. A altura média do primeiro estrato esteve em torno de 17 m e nele foram encontradas algumas espécies típicas como Ocotea lancifolia, Cabralea canjerana, Myrcia fallax, Drimys brasiliensis, Prunus myrtifolia, Lamanonia ternata, Weinmania paulliniifolia, Sapium glandulosum, Myrceugenia alpigena, Myrsine umbellata e Calyptranthes concinna. A altura média do segundo estrato foi de cerca de $11 \mathrm{~m}$, com ocorrência freqüente de Macropeplus dentatus, Meliosma sellowii, Ilex paraguariensis, Croton urucurana e Maytenus evonymoides. A altura média do terceiro estrato esteve em torno de $5 \mathrm{~m}$ e ele é caracterizado pela presença de espécies exclusivas do subbosque, como Miconia cinerascens, Leandra carassana, Dicksonia sellowiana, Symplocos celastrinea e Alsophylla setosa. 
Tabela 1. Espécies arbóreas encontradas no "Matão", município de Camanducaia, Minas Gerais, e exsicata de referência (coletor e número) incluídas nos herbários BHCB e/ou IAC.

Table 1. List of tree species found in the "Matão" area, municipality of Camanducaia, Minas Gerais, Brazil and voucher (collector and number) included in the herbaria BHCB and/or IAC.

Famílias/Espécies

Coletor, $n$.

ANNONACEAE

Rollinia emarginata Schltdl.

Rollinia aff. laurifolia Schltdl.

G.S. França, 142

AQUIFOLIACEAE

Ilex paraguariensis A. St.-Hil.

J.R. Stehmann, 2993

Ilex taubertiana Loes.

G.S. França, 187

ARALIACEAE

Didymopanax angustissimum E. Marchand

Oreopanax fulvum E. Marchand

ASTERACEAE

Baccharis dentata (Vell.) G.M. Barroso

R.B. Torres, 1207

Dasyphyllum flagellare (Casar.) Cabr.

Dasyphyllum sp.

Eupatorium sp.

Pentacalia desiderabilis (Vell.) Cuatrec.

G.S. França, 28

G.S. França, 111

Piptocarpha angustifolia Dusén ex Malme

G.S. França, 99

G.S. França, 71

G.S. França, 288

G.S. França, 281

G.S. França, 105

G.S. França, 30

Senecio macrotis Baker

G.S. França, 106

Vernonanthura ferruginea (Less.) H. Robinson

G.S. França, 238

Vernonanthura puberula (Less.) H. Robinson

G.S. França, 137

CELASTRACEAE

Maytenus evonymoides Reissek

G.S. França, 116

CUNONIACEAE

Lamanonia ternata Vell.

Weinmannia paulliniifolia Pohl

R.B. Torres, 795

CYATHEACEAE

Alsophylla cf. setosa Kalf.

DICKSONIACEAE

Dicksonia sellowiana Hook.

G.S. França, 232

G.S. França, 263

G.S. França, 284

EUPHORBIACEAE

Croton urucurana Baill.

Sapium glandulosum (L.) Morong

G.S. França, 140

FABACEAE

Mimosa scabrella Benth.

J.R. Stehmann, 2500

G.S. França, 127

FLACOURTIACEAE

Casearia sp.

ICACINACEAE

Citronella paniculata (Mart.) Howard

G.S. França, 221

LAURACEAE

Nectandra lanceolata Nees

Nectandra sp.

Ocotea lancifolia $\mathrm{Mez}$

Indeterminada 1

MELASTOMATACEAE

Leandra carassana (DC.) Cogn.

Miconia sp.

Miconia cinerascens Miq.

Miconia pusilliflora (DC.) Naudin

Tibouchina sp.

G.S. França, 136

G.S. França, 286

G.S. França, 249

G.S. França, 192

G.S. França, 115

G.S. França, 196

G.S. França, 108

G.S. França, 261 
MELIACEAE

Cabralea canjerana (Vell.) Mart.

G.S. França, 135

MONIMIACEAE

Macropeplus dentatus (Perkins) I. Santos \& Peixoto

G.S. França, 113

Mollinedia cf. clavigera Tul.

G.S. França, 62

MYRSINACEAE

Myrsine umbellata Mart.

G.S. França, 237

MYRTACEAE

Blepharocalyx salicifolius (Kunth) O. Berg

G.S. França, 54

Calyptranthes concinna DC.

Eugenia pluriflora DC.

Myrceugenia alpigena (DC.) Landrum

Myrceugenia brevipedicellata (Burret) D. Legrand \& Kausel

Myrceugenia glaucescens (Cambess.) D. Legrand \& Kausel

G.S. França, 203

Myrceugenia miersiana (Gardn.) D. Legrand \& Kausel

Myrceugenia myrcioides (Cambess.) O. Berg

Myrcia fallax DC.

Myrcia venulosa DC.

Myrciaria floribunda O. Berg

Pimenta pseudocaryophyllus (Gomes) Landrum

PICRAMNIACEAE

Picramnia gardneri Planch.

R.B. Torres, 991

G.S. França, 248

G.S. França, 126

G.S. França, 290

G.S. França, 224

PODOCARPACEAE

Podocarpus lambertii Klotzsch

R.B. Torres, 1325

PROTEACEAE

Roupala longipetiolata Pohl

G.S. França, 213

G.S. França, 159

ROSACEAE

Prunus myrtifolia Urb.

G.S. França, 264

RUBIACEAE

Alibertia elliptica K. Schum.

G.S. França, 236

Alibertia myrciifolia K. Schum.

G.S. França, 271

Psychotria dusenii Standl.

Psychotria stachyoides Benth.

G.S. França, 268

Psychotria velloziana (Vell.) Muell. Arg.

G.S. França, 190

SABIACEAE

Meliosma sellowii Urb.

G.S. França, 222

SOLANACEAE

Aureliana fasciculata (Vell.) Sendtn.

Solanum caeruleum Vell.

Solanum pseudoquina A. St.-Hil.

Solanum swartzianum Roem. \& Schult.

Solanum sp.

STYRACACEAE

Styrax pohli A. DC.

SYMPLOCACEAE

Symplocos celastrinea Mart. ex Miq.

Symplocos falcata Brand

Symplocos sp.

G.S. França, 246

G.S. França, 279

G.S. França, 141

G.S. França, 274

G.S. França, 199

G.S. França, 131

G.S. França, 206

G.S. França, 270

G.S. França, 211

THEACEAE

Gordonia fruticosa (Schrad.) H. Keng

R.B. Torres, 1286

WINTERACEAE

Drimys brasiliensis Miers

G.S. França, 252 
Tabela 2. Índice de similaridade florística de Jaccard, Ij, calculado utilizando-se o presente levantamento e outras listagens de espécies arbóreas de florestas montanas (sensu lato) do sudeste do Brasil. $\mathrm{A}=$ área amostrada no levantamento, $\mathrm{M}=$ método utilizado na amostragem $(\mathrm{L}=$ levantamento florístico, $\mathrm{P}=$ parcelas $), \mathrm{N}=$ número de espécies arbóreas na listagem; $\mathrm{c}=$ número de espécies em comum com o presente trabalho, H' = índice de diversidade de Shannon.

Table 2. Jaccard's similarity index, Ij, calculated using the present survey and other lists from montane forests (sensu lato) of southeastern Brazil. $\mathrm{A}=$ sample area, $\mathrm{M}=$ method used in the sampling $(\mathrm{L}=$ floristic survey, $\mathrm{P}=$ plots $), \mathrm{N}=$ number of tree species in the list; $\mathrm{c}=$ number of species in common with the present work, $\mathrm{H}^{\prime}=$ Shannon's diversity index.

\begin{tabular}{|c|c|c|c|c|c|c|c|c|}
\hline Local & Referência & $\begin{array}{l}\text { Altitude } \\
\text { (m) }\end{array}$ & A (ha) & M & $\mathrm{N}$ & $\mathrm{c}$ & $\mathrm{Ij}(\%)$ & $\mathrm{H}^{\prime}$ \\
\hline Pq. Est. Ibitipoca, Lima Duarte, $\mathrm{MG}$ & Fontes 1997 & 1.450 & 0,96 & $\mathrm{~L}$ & 199 & 17 & 7,08 & 3,78 \\
\hline Reser. Ecol. Macaé de Cima, Nova Friburgo, RJ & Guedes-Bruni et al. 1997 & 1.100 & 1,00 & $\mathrm{P}$ & 189 & 14 & 6,00 & 4,05 \\
\hline Reser. Biol. Poço Bonito, Lavras, MG & Oliveira Filho et al. 1994a & 1.075 & 0,48 & $\mathrm{P}$ & 119 & 8 & 4,73 & 4,20 \\
\hline Pq. Mun. Grota Funda, Atibaia, SP & Grombone et al. 1990 & 1.150 & 0,42 & $\mathrm{P}$ & 132 & 7 & 3,83 & 4,33 \\
\hline Serra do Japi, Jundiaí, SP & Rodrigues et al. 1989 & 1.020 & 0,42 & $\mathrm{P}$ & 128 & 6 & 3,33 & 4,07 \\
\hline Matão, Camanducaia, MG & O presente trabalho & 1.900 & 0,75 & $\mathrm{P}$ & 58 & - & - & 2,90 \\
\hline
\end{tabular}

Dentre as árvores que atingiram as maiores alturas, citam-se as espécies: Ocotea lancifolia, Cabralea canjerana, Myrcia fallax, Drimys brasiliensis, Myrsine umbellata, Myrceugenia alpigena, Ilex paraguariensis, Lamanonia ternata, Citronella paniculata e Prunus myrtifolia. Aquelas que apresentaram indivíduos em todos os estratos foram: Cabralea canjerana, Myrcia fallax, Drimys brasiliensis, Myrsine umbellata, Myrceugenia alpigena, Calyptranthes concinna e Citronella paniculata. Algumas espécies, como Lamanonia ternata e Weinmannia paulliniifolia, não apresentaram indivíduos jovens no estrato arbóreo.

As espécies com maior IVI foram: Ocotea lancifolia (42,88), Cabralea canjerana $(29,52)$, Psychotria velloziana $(22,95)$, Myrcia fallax $(18,80)$, Drimys brasiliensis $(18,16)$, Myrsine umbellata $(16,60)$ e Myrceugenia alpigena $(9,42)$. Essas sete espécies foram responsáveis por mais de $50 \%$ do IVI total (tabela 3).

O primeiro lugar em importância coube a Ocotea lancifolia, determinado não tanto pelo seu número de indivíduos, mas principalmente por sua grande área basal e elevada dominância relativa. Essa espécie representou $8,3 \%$ de todas as árvores amostradas, mas sua área basal equivale a aproximadamente $30 \%$ do total. As outras espécies apresentaram valores menores de dominância absoluta, dominância relativa e IVI. Em relação à densidade relativa, quatro espécies destacaram-se: Cabralea canjerana, Psychotria velloziana, Myrcia fallax e Drimys brasiliensis, por apresentarem valores maiores do que a espécie citada acima.

As espécies mortas apresentaram um IVI elevado, em torno de 25,87, o que constituiu o terceiro maior valor. Elas representaram $8,26 \%$ de todas as árvores amostradas, e sua área basal foi equivalente a 13,13\% do total.

A análise da freqüência das classes de diâmetro, para a totalidade dos indivíduos amostrados, mostrou um padrão típico de florestas tropicais. A maior concentração dos indivíduos foi encontrada nas classes menores $(10-15 \mathrm{~cm})$, enquanto que nas classes intermediárias o número foi relativamente baixo e nas superiores apresentou-se descontínuo (atingindo até $130 \mathrm{~cm}$ ). Algumas espécies, contudo, não acompanharam esse padrão, como Ocotea lancifolia, que apresentou número reduzido de indivíduos jovens, com menos de $15 \mathrm{~cm}$ de diâmetro.

O quadro comparativo entre os levantamentos florísticos encontra-se na tabela 2. Os resultados das comparações com outras florestas de altitude do sudeste do Brasil mostraram baixa similaridade. As maiores semelhanças $(\mathrm{Ij}>10 \%)$ foram obtidas apenas para os levantamentos realizados no Complexo da Serra da Mantiqueira (Parque Estadual do Ibitipoca, MG) e da Serra do Mar (Reserva Ecológica de Macaé de Cima, RJ). O índice de diversidade de Shannon-Wiener para a mata estudada foi inferior ao de todos os trabalhos comparados. 


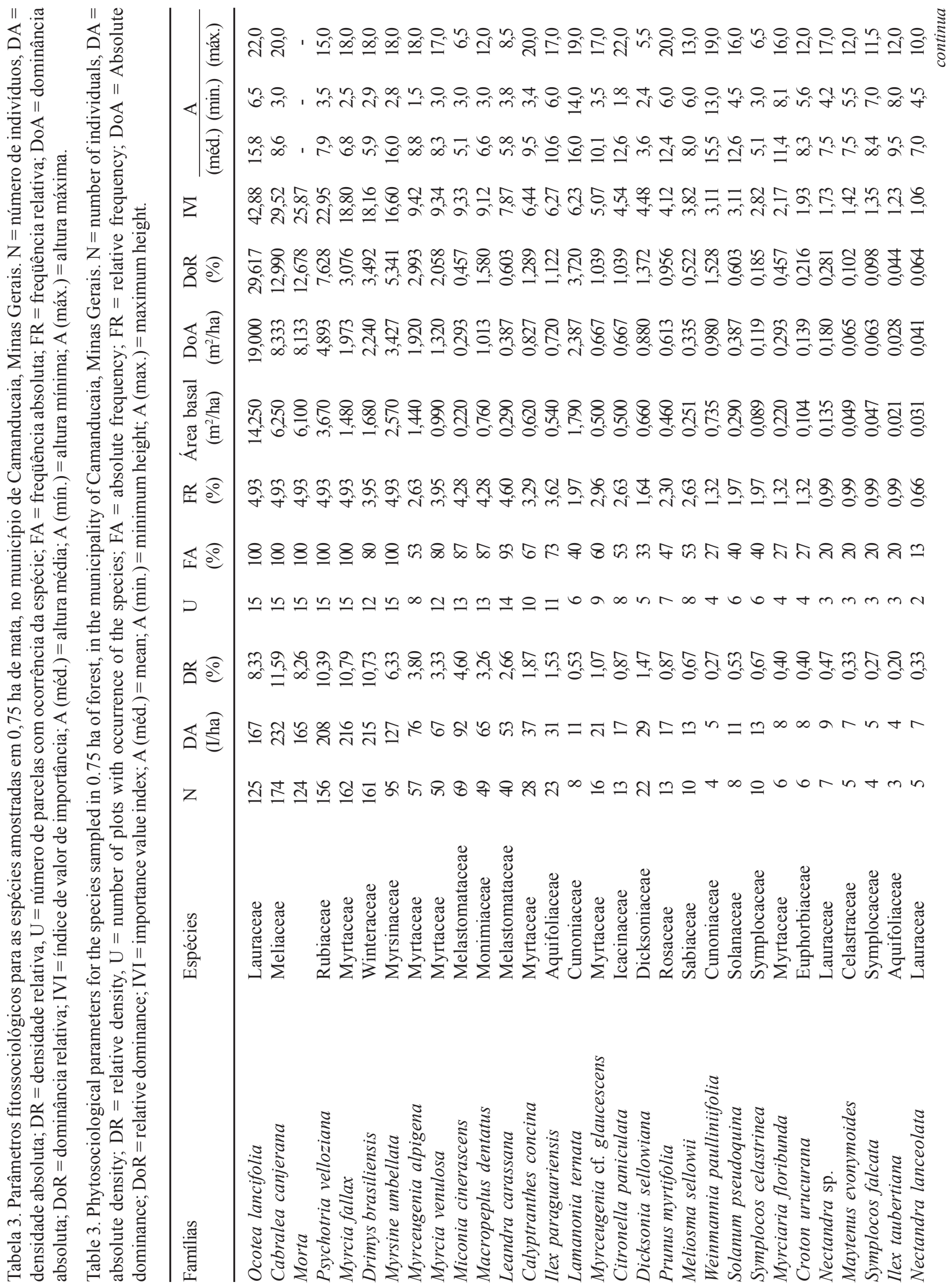




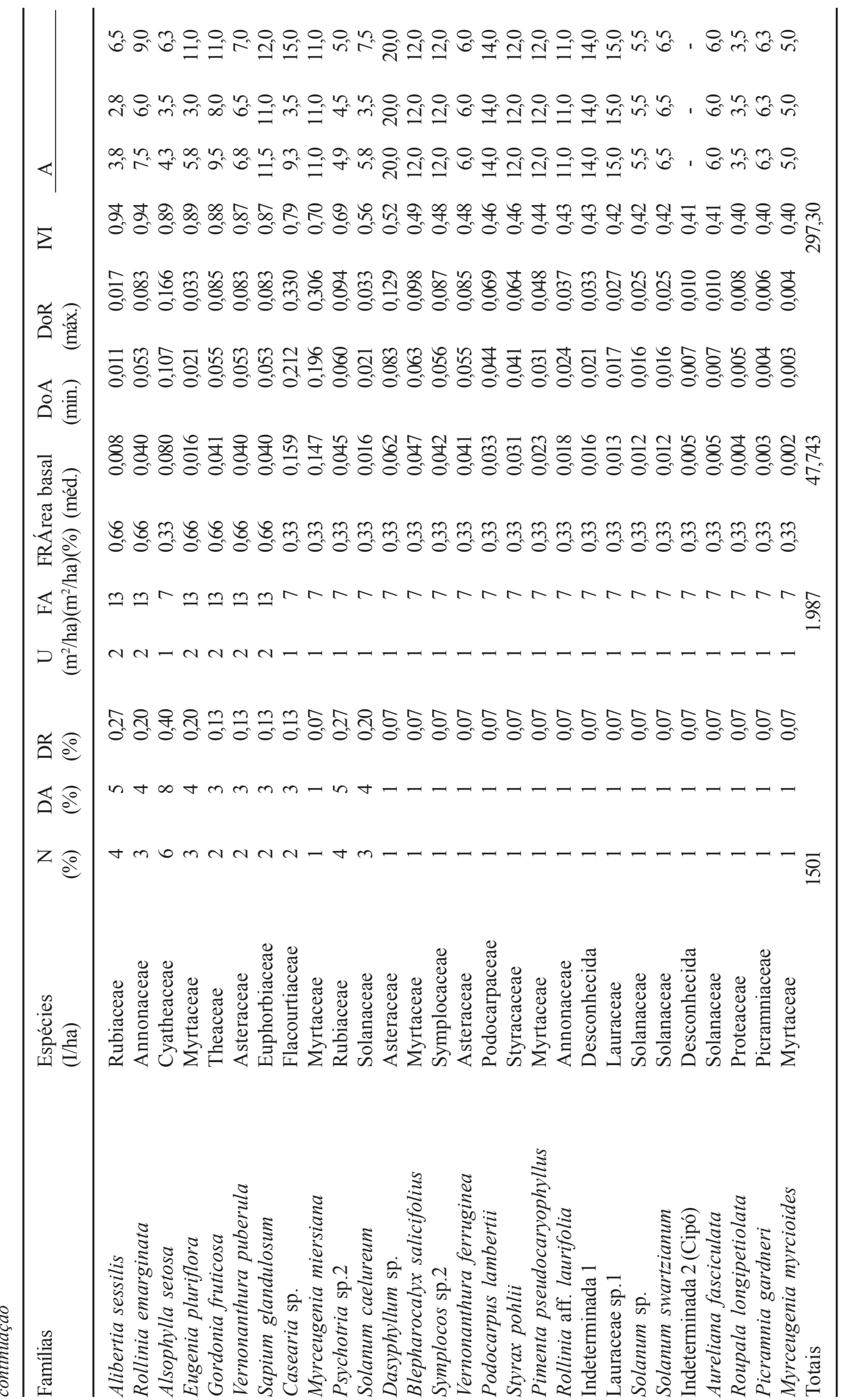




\section{Discussão}

O remanescente estudado constitui vestígio da vegetação arbórea original que cobria os altos dos morros pertencentes ao complexo da Serra da Mantiqueira, que se estendia pelos estados de São Paulo, Minas Gerais e Rio de Janeiro, acompanhando as diferenciações climáticas e de altitude (Azevedo 1962). Devido à ocupação humana nessas regiões, a grande extensão de floresta foi reduzida a vários remanescentes, localizados nas áreas mais elevadas e de difícil exploração, mas que provavelmente ainda guardam características da floresta que existiu no passado.

Nas cadeias montanhosas da região neotropical, as florestas nebulares ocorrem entre 1.000 e $3.000 \mathrm{~m}$, mas há consideráveis variações locais e regionais nos limites altitudinais (Webster 1995). Para a Serra do Itatiaia, Segadas-Vianna (1968) sugeriu que acima de $2.000 \mathrm{~m}$ de altitude, o clima rigoroso, com invernos frios e secos, limita o estabelecimento de vegetação arbórea na maioria dos hábitats. Assim, a mata estudada provavelmente se encontra um pouco abaixo da altitude que limita a dominância da vegetação florestal na Serra da Mantiqueira.

Em relação aos solos verifica-se que os Latossolos Vermelho Amarelos observados são muito expressivos no domínio pedobioclimático do "Mar de Morros Florestados" (Resende 1995), paisagem encontrada na região dos municípios de Camanducaia e Gonçalves. $\mathrm{Na}$ área da mata estudada, os solos são bastante ácidos e há grande quantidade de matéria orgânica, chegando a ser turfoso em alguns locais, sendo portanto classificados como Organossolos. Isso é decorrente da decomposição restrita de "liter"(e conseqüente acúmulo de matéria orgânica), causado principalmente pelo excesso de água e drenagem pobre sob baixas temperaturas (Resende 1995, Carvalho et al. 2000).

Nas florestas andinas, as famílias Lauraceae, Melastomataceae e Rubiaceae possuem maior riqueza de espécies lenhosas em altitudes acima de 1.500 m s.n.m. (Gentry 1995). A grande importância relativa de algumas famílias, como Myrtaceae, Lauraceae, Meliaceae, Rubiaceae, Winteraceae, Melastomataceae, Myrsinaceae e Monimiaceae, encontradas neste trabalho, é característica das florestas localizadas acima de $1.000 \mathrm{~m}$ de altitude no sudeste do Brasil (Fontes 1997). A ausência de Fabaceae na área de estudo reflete o padrão de riqueza florística conhecido para a família, que decresce com o aumento da altitude (Gentry 1995, Oliveira Filho 2000).
Em termos florísticos, a floresta estudada pode ser comparada às comunidades arbóreas das regiões elevadas da Mantiqueira, do planalto de Poços de Caldas e do maciço do Itatiaia, indicando Podocarpus lambertii e espécies dos gêneros Nectandra e Ocotea como típicas do estrato arbóreo (Azevedo 1962). Para a região do Itatiaia, estas matas são descritas ainda como vegetação do nível montano médio (1.700 até $2.000 \mathrm{~m}$ ), de 20 a $30 \mathrm{~m}$ de altura, dominadas pelo gênero Cabralea (Segadas-Vianna 1968). O estrato inferior é ocupado por árvores dos gêneros: Roupala, Drimys e Weinmannia. Samambaias arbóreas são comuns, geralmente formando colônias compactas. Tais matas corresponderiam à Floresta Ombrófila Densa Montana (Mata Alta), fortemente relacionada ao tipo do relevo (vales e depressões) e ao acúmulo de sedimentos e umidade (Lima \& Guedes-Bruni 1997).

Os gêneros Drimys, Leandra, Psychotria, Croton e Weinmannia, especialmente Cabralea (representado por Cabralea canjerana), tiveram grande dominância, sendo observados também nas florestas montanas do Itatiaia (Segadas-Vianna \& Dau 1968). A importância relativa de alguns gêneros pode aumentar com a altitude. Foram encontrados no levantamento florístico gêneros que se destacaram por serem fortemente relacionados a altitudes elevadas: Drimys, Weinmannia, Podocarpus, Meliosma, Ilex, Myrsine, Miconia, Prunus e Roupala (Mainieri \& Pires 1973, Webster 1995, Fontes 1997, Oliveira Filho \& Fontes 2000). Ocorreram também espécies indicadoras de altitude: Drimys brasiliensis, Weinmannia paulliniifolia, Podocarpus lambertii, Gordonia fruticosa, Meliosma sellowii, Nectandra lanceolata, Pimenta pseudocayophyllus e Symplocos celastrinea (Oliveira Filho \& Fontes 2000).

A espécie com maior importância no estudo fitossociológico foi Ocotea lancifolia (Lauraceae). É citada como ocorrente na região Sudeste do Brasil (MG e SP) (Coe-Teixeira 1980). Em Minas Gerais, foi registrada para a Cadeia do Espinhaço, nas matas ripárias de altitude (1.200 m s.n.m) da Serra do Cipó (Meguro et al. 1996).

As espécies mortas obtiveram o terceiro lugar em importância, determinado principalmente por sua densidade absoluta e por sua área basal. Elas representaram $8,26 \%$ de todas as árvores amostradas. Esse valor pode ser considerado normal dentro das comunidades florestais que é estimado entre $2,7 \%$ e $10 \%$ da fitomassa viva (Martins 1991). A ocorrência de muitas árvores mortas no interior da floresta pode ser tanto uma decorrência de morte natural, como conseqüência de acidentes (ventos, tempestades, queda 
de galhos grandes, etc.), parasitismo, ou, ainda, decorrente de perturbações introduzidas pelo homem num passado recente (Martins 1991).

As florestas tropicais pluviais geralmente apresentam três estratos arbóreos (inferior, médio e dossel) e espécies emergentes (Richards 1976). Estes estratos foram observados na mata estudada, apesar de dois deles (médio e dossel) serem pouco diferenciados. As espécies emergentes não foram evidenciadas, visto que o dossel da mata apresentou-se contínuo com alturas semelhantes. A não evidência de alguns estratos arbóreos mais demarcados decorre da diminuição da altura das árvores com o aumento da altitude. Isso, provavelmente, é determinado por fatores que limitam o crescimento dos indivíduos como: a competição pela luz em vales profundos e estreitos, a maior irradiação solar em áreas abertas e o déficit hídrico sasonal, verificado por exemplo em regiões de cordilheiras (Oliveira Filho et al. 1994c). Outros fatores como a freqüencia de geadas, a saturação, acidez e baixa fertilidade do solo também contribuem para determinar as características estruturais das florestas montanas (Bruijnzell \& Veneklaas 1998). Algumas espécies encontradas no estrato inferior, como Miconia cinerascens, Leandra carassana, Dicksonia sellowiana, Symplocos celastrinea e Alsophylla setosa, também foram citadas para o sub-bosque de outras florestas montanas (Meira Neto et al. 1989, Grombone et al. 1990, Oliveira Filho \& Machado 1993, Carvalho et al. 1995, Fontes 1997). Várias espécies encontradas no estrato superior, tais como Cabralea canjerana, Weinmannia paulliniifolia, Lamanonia ternata, Ilex paraguariensis, Citronella paniculata, Myrsine umbellata, Drimys brasiliensis, Calyptranthes concinna e Solanum pseudoquina foram registradas para o dossel da mata em alguns trabalhos com florestas montanas (Oliveira Filho et al. 1994 a, b, c, Guedes-Bruni et al. 1997, Carvalho et al. 2000).

As espécies do estrato médio e superior que se destacaram pelo seu maior valor de importância parecem estar se regenerando na mata, pois foram encontrados indivíduos ocupando o dossel e o sub-bosque. Apenas duas espécies estiveram ausentes dos estratos inferiores, Weinmannia paulliniifolia e Lamanonia ternata, que podem constituir espécies do passado dessa floresta, e que hoje são observadas predominantemente no estrato superior.

O índice de diversidade encontrado foi menor do que aqueles obtidos para outras florestas montanas do sudeste do Brasil. Tal fato, provavelmente, se relaciona à elevada altitude. Uma correlação altamente significativa em relação à diminuição da diversidade de espécies com o aumento da altitude (acima de $1.500 \mathrm{~m}$ s.n.m.) foi verificada para as florestas montanas dos Andes (Gentry 1995). A baixa diversidade pode estar relacionada às condições climáticas influenciadas pela altitude elevada. O clima frio e seco nos meses de julho e agosto, a presença de geadas e a alta umidade nos meses de chuva, provavelmente limitariam o estabelecimento de algumas espécies (Oliveira Filho \& Fontes 2000). O fato da área estudada estar situada numa região de "Mar de Morros Florestados", mais especificamente no topo de um desses morros, também poderia estar influenciando a diversidade, pois faltam os gradientes de declividade que poderiam contribuir com espécies para a comunidade, como ocorre em áreas de encosta.

O remanescente estudado apresentou baixa similaridade com outras florestas montanas do sudeste do Brasil, provavelmente por situar-se em altitude mais elevada (1.900 m s.n.m.). Apesar disso, a maior semelhança dentre as áreas comparadas ocorreu com a flora do Parque Estadual do Ibitipoca (Fontes 1997), compartilhando 17 espécies do total de 199 listadas, dentre elas Cabralea canjerana, Myrsine umbellata, Drimys brasiliensis e Solanum pseudoquina. Tal semelhança entre áreas, mesmo que pequena $(<8 \%)$, deve-se geralmente à maior proximidade geográfica, na medida que reflete maiores semelhanças ambientais, sobretudo climáticas (Oliveira Filho \& Machado 1993) e de altitude, visto ser este fator relevante na diferenciação da florística arbórea (Fontes 1997, Oliveira Filho \& Fontes 2000).

Diversos fatores conferem ao remanescente estudado uma grande relevância ambiental: a sua função de mantenedor das nascentes que abastecem diversos mananciais da região; o seu tamanho por ser a maior área de floresta nativa na região, o seu papel relitual para espécies típicas de florestas de altitude e a presença de espécie ameaçada de extinção (Dicksonia sellowiana). Considerando esses fatores e que a paisagem regional foi muito alterada, especialmente pelo reflorestamento com espécies exóticas e culturas anuais, a proteção deste remanescente é de fundamental importância para a conservação da biodiversidade da floresta atlântica na região sul de Minas Gerais.

Agradecimentos - Ao Altair Rezende de Souza, por permitir o desenvolvimento dos trabalhos em sua propriedade; ao Toninho Buta, pelo apoio logístico no trabalho de campo; aos especialistas que contribuíram na identificação das espécies: Alexandre Salino (Pteridófitas), H. Robinson 
(Asteraceae), Henk Van der Werff (Lauraceae), João Semir (Asteraceae), Marcos Sobral (Myrtaceae), Maria Lúcia Kawasaki (Myrtaceae); à Luciana Y. Kamino e ao Rubens Custódio da Mota, pelo auxílio na coleta de dados. Ao CNPq, pela bolsa de produtividade em pesquisa para J.R. Stehmann. Por fim, à Edivani V. Franceschinelli, coordenadora do projeto "Estudos de Conservação e Recuperação dos Fragmentos Florestais da APA do Camanducaia, MMA/Probio", pelo apoio e estímulo dispensados.

\section{Referências bibliográficas}

AZEVEDO, L.G. 1962. Tipos de vegetação do sul de Minas e campos da Mantiqueira (Brasil). Anais da Academia Brasileira de Ciências 34:225-234.

BRUIJNZEEL, L.A. \& VENEKLAAS, E.J. 1998. Climatic conditions and tropical montane forest productivity: the fog has not lifted yet. Ecology 79:3-9.

CARVALHO, D.A., OLIVEIRA FILHO, A.T., VILELA, E.A. \& GAVILANES, M.L. 1995. Flora arbustivo-arbórea de uma floresta ripária no alto rio Grande em Bom Sucesso/MG. Acta Botanica Brasilica 9:231-245.

CARVALHO, L M.T., FONTES, M.A.L. \& OLIVEIRA FILHO, A.T. 2000. Tree species distribution in canopy gaps and mature forest in an area of cloud forest of the Ibitipoca Range, south-eastern Brazil. Plant Ecology 149:9-22.

COE-TEIXEIRA, B. 1980. Lauraceae do gênero Ocotea, do Estado de São Paulo. Rodriguésia 32:55-190.

COSTA, C.M.R., HERRMANN, G., MARTINS, C.S., LINS, L.V. \& LAMAS, I.R. (orgs.). 1998. Biodiversidade em Minas Gerais. Um atlas para sua conservação. Fundação Biodiversitas, Belo Horizonte.

EMBRAPA. 1981. Mapa de solos do Brasil, 1:5.000.000. Empresa Brasileira de Pesquisa Agropecuária, Rio de Janeiro.

EMBRAPA. 1999. Sistema brasileiro de classificação de solos. Empresa Brasileira de Pesquisa Agropecuária, Centro Nacional de Pesquisa de Solos, Rio de Janeiro.

FALKENBERG, D.B. \& VOLTOLINI, J.C. 1995. The montane cloud forest in Southern Brazil. In Tropical montane cloud forests (L.S. Hamilton, J.O. Juvik \& F.N. Scatena, eds.). Springer-Verlag, New York, p.138-149.

FIDALGO, O. \& BONONI, V.L.R. 1984. Técnicas de coleta, preservação e herborização de material botânico. Manual 4. Instituto de Botânica, São Paulo.

FONTES, M.A.L. 1997. Análise da composição florística das florestas nebulares do Parque Estadual do Ibitipoca, Minas Gerais, Brasil. Dissertação de mestrado, Universidade Federal de Lavras, Lavras.

GENTRY, A.H. 1995. Patterns of diversity and floristic composition in neotropical montane forests. In Biodiversity and conservation of Neotropical montane forests (S.P. Churchill, H. Balslev, E. Forero \& J.L. Luteyn, eds.). The New York Botanical Garden, New York, p.103-126.
GROMBONE, M.T., BERNACCI, L.C., MEIRA NETO, J.A.A., TAMASHIRO, J.Y. \& LEITÃO FILHO, H.F. 1990. Estrutura fitossociológica da floresta semidecídua de altitude do Parque Municipal da Grota Funda, Atibaia - Estado de São Paulo. Acta Botanica Brasilica 4:47-64.

GUEDES-BRUNI, R.R., PESSOA, S.V.A. \& KURTZ, B.C. 1997. Florística e estrutura do componente arbustivo-arbóreo de um trecho preservado de floresta montana na Reserva Ecológica de Macaé de Cima. In Serra de Macaé de Cima: diversidade florística e conservação em Mata Atlântica (H.C. Lima \& R.R. Guedes-Bruni, eds.). Jardim Botânico do Rio de Janeiro, Rio de Janeiro, p.127-167.

INMET. 1982. 5 Distrito de Meteorologia. Zoneamento Climatológico de Minas Gerais. Classificação Climática de Köeppen / Set. Instituto Nacional de Meteorologia, Belo Horizonte.

LIMA, H.C. \& GUEDES-BRUNI, R.R. 1997. Plantas arbóreas da Reserva Ecológica de Macaé de Cima. In Serra de Macaé de Cima: diversidade florística e conservação em Mata Atlântica (H.C. Lima \& R.R. Guedes-Bruni, eds.). Jardim Botânico do Rio de Janeiro, Rio de Janeiro, p.53-63.

MAGURRAN, A. 1988a. BIODAP. Ecological diversity and its measurement. Resource Conservation Fundy National Park, Alma.

MAGURRAN, A.E. 1988b. Ecological diversity and its measurement. Princeton University Press, Princeton.

MAINIERI, C. \& PIRES, J.M. 1973. O gênero Podocarpus no Brasil. Silvicultura 8:1-24.

MARTINS, F.R. 1991. Estrutura de uma floresta mesófila. Universidade Estadual de Campinas, Campinas.

MEGURO, M., PIRANI, J.R., MELLO SILVA, R. \& GIULIETTI, A.M. 1996. Caracterização florística e estrutural de matas ripárias e capões de altitude da Serra do Cipó, Minas Gerais. Boletim de Botânica da Universidade de São Paulo 15:13-29.

MEIRA NETO, J.A.A., BERNACCI, L.C., GROMBONE, M.T., TAMASHIRO, J.Y. \& LEITÃO FILHO, H.F. 1989. Composição florística da floresta semidecídua de altitude do Parque Municipal da Grota Funda, Atibaia, Estado de São Paulo. Acta Botanica Brasilica 3:51-74.

OLIVEIRA, J.B. 1992. Classes gerais de solos do Brasil: guia auxiliar para seu reconhecimento. Funep, Unesp, São Paulo.

OLIVEIRA FILHO, A.T. \& MACHADO, J.N.M. 1993. Composição florística de uma floresta semidecídua montana, na Serra de São José, Tiradentes Minas Gerais. Acta Botanica Brasilica 7:71-88.

OLIVEIRA FILHO, A.T., ALMEIDA, R.J., MELLO, J.M. \& GAVILANES, M.L. 1994a. Estrutura fitossociológica e variáveis ambientais em um trecho da mata ciliar do córrego dos Vilas Boas, Reserva Biológica do Poço Bonito, Lavras (MG). Revista Brasileira de Botânica 17:67-85. 
OLIVEIRA FILHO, A.T., SCOLFORO, J.R.S. \& MELLO, J.M. 1994b. Composição florística e estrutura comunitária de um remanescente de floresta semidecídua montana em Lavras, MG. Revista Brasileira de Botânica 2:167-182.

OLIVEIRA FILHO, A.T., VILELA, E.A., CARVALHO, D.A. \& GAVILANES, M. 1994c. Differentiation of streamside and upland vegetation in an area of montane semideciduous forest in southeastern Brazil. Flora 189:287-305.

OLIVEIRA FILHO A.T. \& FONTES, M.A.L. 2000. Patterns of floristic differentiation among Atlantic Forests in Southeastern Brazil and the influence of climate. Biotropica 32:793-810.

PAGANO, S.N. \& LEITÃO FILHO, H.F. 1987. Composição florística do estrato arbóreo de mata mesófila semidecídua no município de Rio Claro, estado de São Paulo. Revista Brasileira de Botânica 10:37-48.

RESENDE, M. 1995. Pedologia: base para distinção de ambientes. Neput, Viçosa.

RICHARDS, P.W. 1976. The tropical rain forest, an ecological study. Cambridge University Press, New York.

RODRIGUES, R.R., MORELLATO, L.P.C., JOLY, C.A. \& LEITÃO FILHO, H.F. 1989. Estudo florístico e fitossociológico em um gradiente altitudinal de mata estacional mesófila semidecídua, na Serra do Japi, Jundiaí, SP. Revista Brasileira de Botânica 12:71-84.
SEGADAS-VIANNA, F. 1968. Ecology of the Itatiaia Range, Southeastern Brazil I. Altitudinal zonation of the vegetation. Arquivos do Museu Nacional 53:7-30.

SEGADAS-VIANNA, F. \& DAU, L. 1968. Ecology of the Itatiaia Range, Southeastern Brazil II. Climates and altitudinal climatic zonation. Arquivos do Museu Nacional 53:31-53.

SHEPHERD, G.J. 1994. Fitopac: manual do usuário. Universidade de Campinas, Campinas.

VELOSO, H.P., RANGEL FILHO, A.L. \& ALVES-LIMA, J.C. 1991. Classificação da vegetação brasileira, adaptada a um sistema universal. Instituto Brasileiro de Geografia e Estatística, Rio de Janeiro.

WALTER, H. 1986. Vegetação e zonas climáticas. Tratado de Ecologia Global. Editora Pedagógica e Universitária, São Paulo.

WEBSTER, G.L. 1995. The panorama of Neotropical cloud forests. In Biodiversity and conservation of Neotropical montane forests (S.P. Churchill, H. Balslev, E. Forero \& J.L. Luteyn, eds.). The New York Botanical Garden, New York, p.53-77.

WHITMORE, T.C. 1990. An introduction to tropical rain forests. Clarendon Press, Oxford. 